\title{
Visible Light Induced Knoevenagel Condensation Catalyzed by Starfruit Juice of Averrhoa carambola
}

\author{
Rammohan Pal*, Taradas Sarkar \\ Department of Chemistry, Acharya Jagadish Chandra Bose College, Kolkata, India \\ Email: ${ }^{*}$ pal rammohan@yahoo.com
}

Received 7 April 2014; revised 12 May 2014; accepted 19 May 2014

Copyright (C) 2014 by authors and Scientific Research Publishing Inc. This work is licensed under the Creative Commons Attribution International License (CC BY). http://creativecommons.org/licenses/by/4.0/ c) (i) Open Access

\begin{abstract}
Aqueous starfruit juice catalyzed a simple and efficient Knoevenagel condensation of aromatic aldehydes with malononitrile has been developed under visible light. Products were obtained in yields up to $\mathbf{9 8 \%}$ after short reaction times and they were isolated by simple filtration in pure crystallization states. The method is green and economically viable. A plausible mechanism for photochemical Knoevenagel condensation reaction catalyzed by starfruit juice was also predicted.
\end{abstract}

\section{Keywords}

Knoevenagel Condensation, Aqueous Starfruit Juice, Aldehydes, Malononitrile, Visible Light

\section{Introduction}

Knoevenagel condensation, first demonstrated by Emil Knoevenagel in 1894 [1], is one of the most important and widely employed methods for carbon-carbon double bond formation in synthetic chemistry [2]-[4]. It has been used for the preparation of a wide range of substituted electrophilic alkenes, and for the synthesis of intermediate such as coumarin derivatives which are useful in perfumes, cosmetics and bioactive compounds [5]-[8]. In addition, Knoevenagel condensation products exhibit inhibition of antiphosphorylation of EGF-receptor and antiproliferative activity [9]. As a result of their importance from a pharmacological, industrial and synthetic point of view a large number of methods for the Knoevenagel condensation have been reported using various Lewis bases/acids [10]-[14]. The use of Green Chemistry protocol based on microwave assisted reaction [15][20], ultrasound irradiation [21] [22], biotechnology-based approach [23] [24], solid phase [25] [26], green solvent like ionic liquid [27]-[33] or water [34]-[36], and grindstone method under solvent-free condition [37]-[39]

*Corresponding author.

How to cite this paper: Pal, R. and Sarkar, T. (2014) Visible Light Induced Knoevenagel Condensation Catalyzed by Starfruit Juice of Averrhoa carambola. International Journal of Organic Chemistry, 4, 106-115.

http://dx.doi.org/10.4236/ijoc.2014.42012 
have also been developed. It is noteworthy to observe that all these protocols have some drawbacks, such as use of expensive catalyst, high thermal conditions, disposal of toxic solvents and catalyst, long reaction time often pose a problem.

In the past two decades, classical organic chemistry had been rewritten around new approaches that search for products and processes in the chemical industry that are environmentally acceptable [40]. Therefore, to address depletion of natural resources and preservation of ecosystem is just urgent to develop so called "greener technologies" to make chemical agents for well being of human health [41].

An attractive area in organic synthesis involves photochemical reactions particularly using visible light in environment-friendly solvent like water or aqueous ethanol and is generally considered as a clean and green procedure. This type of photo-activation of substrate very often minimizing the formation of by-products and for this reason, photochemical reactions occupy an interesting position and excellent reviews/paper have been published [42]-[47]. The use of water as a reaction medium is not only inexpensive and environmentally benign but also provides completely different reactivity [48]. It has been suggested that the effect of water on organic reaction may be due to the high internal pressure exerted by a water solution which results from the high cohesive energy of water [49].

A number of organic reactions using natural catalysts such as clay [50]-[53], natural phosphate [54] [56], animal bone [57], and also various fruit juice's are reported in literature. Due to acidic nature, aqueous fruit juice like lemon [58]-[64], pineapple [65] [66], coconut [67], Acacia concinna [68], Sapindus trifolistus [69] and Tamarindus indica [70] [71] fruit has been found to be a suitable replacement for various homogeneous acid catalysis. In accordance with this, we report the Knoevenagel condensation of aromatic aldehydes with malononitrile in presence of aqueous starfruit juice, a natural, green and biocatalyst system stimulated by visible light.

Starfruit (Averrhoa carambola) (Figure 1) is grown extensively in Philippines, Indonesia, Malaysia, India, Bangladesh, Latin America and Sri Lanka. It has long been one of the most popular of the citrus tropical and subtropical fruits, largely because of its attractive flavor and refreshing sugar-acid balance. Starfruit juice of Averrhoa carambola shows antioxidant properties due to scavenging of nitric oxide (NO) and antimicrobial activities against E. coli, Klebsiella spp. and Staphylococcus aureus [72].

The main ingredients [73] [74] of $100 \mathrm{gm}$ of unripe starfruit contain water (89 - $91 \mathrm{~g})$, protein (0.38 g), fat (0.08 g), carbohydrates (9.38 g), sugars (3.98 g), edible fiber (0.8 - $0.9 \mathrm{~g}$ ), calcium (4.4 - $6.0 \mathrm{mg}$ ), phosphorous (15.5 - $21 \mathrm{mg})$, sodium (2 mg) and potassium (133 mg). Fresh mature unripe fruit were found to have a total acid content of $12.51 \mathrm{mg}$ consisting of $5 \mathrm{mg}$ oxalic acid, $4.37 \mathrm{mg}$ tartaric acid, $1.32 \mathrm{mg}$ citric acid, $1.21 \mathrm{mg}$ malic acid, $0.22 \mathrm{mg}$ succinic acid, $0.26-0.53 \mathrm{mg}$ ascorbic acid and $0.39 \mathrm{mg}$ pantothenic acid. The composition of the starfruit juice varies with geographical, cultural and seasonal harvesting and processing. An aqueous extract of starfruit juice is acidic due to presence of edible organic acids and hence it will be work as an acid catalyst for acid catalyzed reactions.

\section{Results and Discussion}

In continuation of our research interest concerning the investigation of new natural catalyst and development of

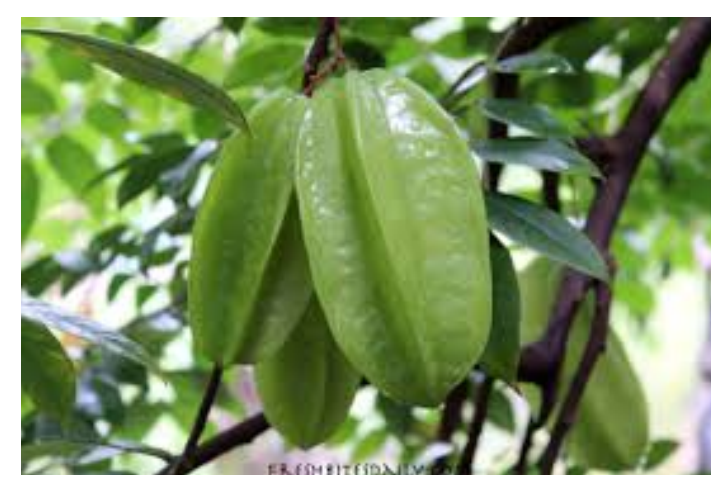

Figure 1. Photoghrapy of starfruit of Averrhoa carambola. 
new methodologies $(62-64,70,71)$ herein is reported in this paper, for the first time a highly efficient, ecofriendly and economic method for Knoevenagel condensation of aldehydes with malononitrile using aqueous starfruit juice stimulated by visible light affording 2-(substituted phenylidene) malononitrile (Scheme 1).

The photochemical reactions were found to be very clean and the products were obtained in extremely pure crystalline states with an average yield of $75 \%$ - 98\% and the reaction time varied on an average 2 - 7 min. The products were isolated from the reaction mixture in pure crystalline form by cooling in an ice-bath and need no further crystallization for aromatic aldehydes and the results are given in Table 1.

The scope of application of the presented method is demonstrated by using various substituted aromatic aldehydes to react with malononitrile. The procedure was successfully applied for heteroaromatic aldehydes (entries 18,19 ), and the ether (entries $3,9,15,16$ ), esters (entries 14,15 ) linkages in the aromatic aldehydes were unaffected under photochemical conditions. The reaction was further explored for the synthesis of $p$-bis-2(phenylidene) malononitrile (3t) in 95\% yield by the condensation of terephthalaldehyde (entry 20) with two mole of malononitrile under similar reaction conditions.

When the same reactions were performed at room temperature for $1 \mathrm{~h}$, only $30 \%-35 \%$ of the corresponding products were isolated. On the hand, under refluxing conditions for 10 min only $30 \%-40 \%$ of 3 were isolated and the yield of the products increased to $60 \%-65 \%$ after $3-4 \mathrm{~h}$. The microwave irradiation reaction, accomplished in an average time periods 2 - 3 min. In all the above cases products were isolated by column chromatography or required further crystallization from appropriate solvents. Thus, the present method in comparison with room temperature, thermal and microwave irradiation one, is encouragingly effectual and smoothly for aromatic aldehydes free from any adhering by byproduct or side products.

In the present instance, we speculate that the reaction may plausibly be initiated by homolytic C-H bond cleavage of malononitrile (2) in the presence of light to produce a radical I and hydrogen radical which is immediately converted to a transient anion radical II due to a weak interaction with water molecules. Aldehyde (1) becomes activated by protonation from starfruit juice to produce a protonated species III. One electron transfer from II to IV produced a radical V, which couples with radical I to form VI. Protonation of VI followed by dehydration from VII to form the title compound $\mathbf{3}$ as depicted in Scheme 2.

All the products (Table 1) are known compounds and their structures are confirmed by comparison of melting

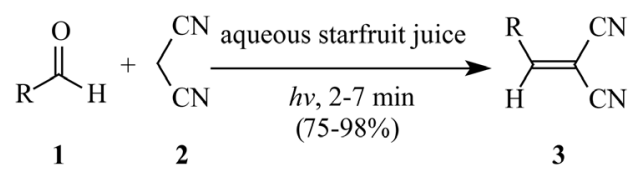

$\mathrm{R}=$ aryl, cinnamyl, heteroaryl $\quad$ (20 examples)

Scheme 1. Visible light induced Knoevenagel condensation reaction catalyzed by starfruit juice.

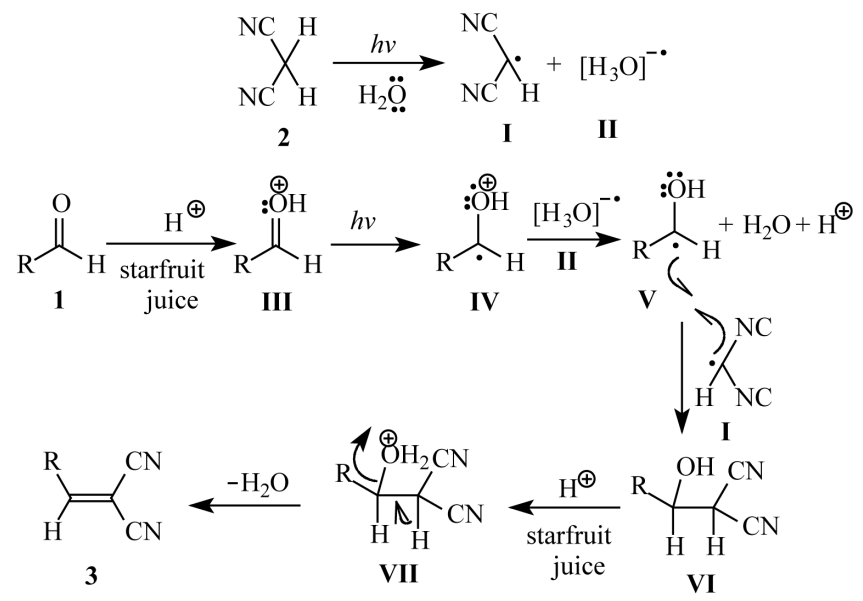

Scheme 2. Plausible mechanistic pathway for the photochemical Knoevenagel condensation of aldehydes and malononitrile catalyzed by starfruit juice. 
Table 1. Results of photochemical Knoevenagel condensation of aldehydes with malononitrile catalyzed by aqueous starfruit juice.

\begin{tabular}{|c|c|c|c|c|c|}
\hline Entry & Substrate (1) & Product (3) ${ }^{\mathrm{a}}$ & Yield (\%) ${ }^{\mathrm{b}}$ & Time (min) & M.p. $\left({ }^{\circ} \mathrm{C}\right)[\text { Lit. Value }]^{\mathrm{c}}$ \\
\hline 1 & & $\begin{array}{l}\mathrm{NC} \\
\mathbf{3 a}\end{array}$ & 92 & 3 & $\begin{array}{c}81-82 \\
{[80]-[83]^{37}}\end{array}$ \\
\hline 2 & $1 b$ & $3 \mathbf{b}$ & 94 & 4 & $\begin{array}{c}133-134 \\
{[134][135]^{27}}\end{array}$ \\
\hline 3 & & 3c & 85 & 5 & $\begin{array}{c}113-115 \\
{[116]^{66}}\end{array}$ \\
\hline 4 & 1d & & 75 & 4 & $\begin{array}{c}160 \\
{[159]^{66}}\end{array}$ \\
\hline 5 & $1 e$ & $3 e$ & 95 & 2 & $\begin{array}{c}165 \\
{[164]^{71}}\end{array}$ \\
\hline 6 & 1f & $3 f$ & 82 & 4 & $\begin{array}{c}182-184 \\
{[185][186]^{20}}\end{array}$ \\
\hline 7 & $1 \mathrm{~g}$ & & 93 & 4 & $\begin{array}{c}164-166 \\
{[162]-[164]^{19}}\end{array}$ \\
\hline 8 & 1h & & 80 & 7 & $\begin{array}{c}158 \\
{[156]^{66}}\end{array}$ \\
\hline 9 & 1i & $3 \mathbf{i}$ & 92 & 6 & $\begin{array}{c}132-134 \\
{[135][136]^{20}}\end{array}$ \\
\hline 10 & $\mathbf{1 j}$ & $3 \mathbf{j}$ & 90 & 5 & $\begin{array}{c}182 \\
{[180]^{22 b}}\end{array}$ \\
\hline
\end{tabular}




\section{Continued}

11<smiles>O=Cc1ccccc1[N+](=O)[O-]</smiles>

$1 k$

12<smiles>O=Cc1cccc([N+](=O)[O-])c1</smiles>

11

13<smiles>O=Cc1ccc([N+](=O)[O-])cc1</smiles>

14<smiles>O=Cc1ccc(OC(=O)c2ccccc2)cc1</smiles>

15<smiles>COc1cc(C=O)ccc1OC(=O)c1ccccc1</smiles>

10

16<smiles>O=Cc1ccc2c(c1)OCO2</smiles>

1p

17

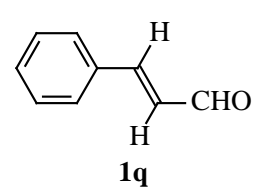

18

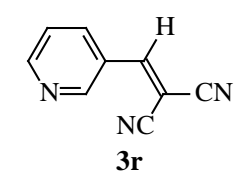

19<smiles>Cc1[nH]c2ccccc2c1C(c1ccc(C=O)cc1)c1c(C)[nH]c2ccccc12</smiles>

$1 \mathrm{~s}$<smiles>N#CC(C#N)=Cc1ccccc1[N+](=O)[O-]</smiles>

3k<smiles>N#CC(C#N)=Cc1cccc([N+](=O)[O-])c1</smiles>

31
95

$$
3
$$

134

[136]-[138 $]^{27}$<smiles>N#CC(C#N)=Cc1ccc([N+](=O)[O-])cc1</smiles>

90<smiles>N#CC(C#N)=Cc1ccc(OC(=O)c2ccccc2)cc1</smiles>

94

3n<smiles>COc1cc(C=C(C#N)C#N)ccc1OC(=O)c1ccccc1</smiles>

92

30<smiles>N#CC(C#N)=Cc1ccc2c(c1)OCO2</smiles>

98

78<smiles>N#CC(C#N)=C/C=C/c1ccccc1</smiles>

$3 q$<smiles>N#C/C(Br)=C/c1cccnc1</smiles>

90<smiles>Cc1[nH]c2ccccc2c1C(c1ccc(C=C(C#N)C#N)cc1)c1c(C)[nH]c2ccccc12</smiles>

3s<smiles>N#CC(C#N)=Cc1ccc(C=C(C#N)C#N)cc1</smiles>

4

$[198]^{11}$
152 - 154

$[152]^{71}$ $[140][141]^{71}$

$104-106$ [107] $[108]^{20}$

161 - 163 [160]-[162 $]^{19}$

2

7

7

125 - 127 [127]-[129] ${ }^{37}$

80 - 82 [84] $[85]^{27}$

78

7

320 - 322

$[320]-[322]^{71}$

95

3

300 $[298]-[300]^{71}$

20

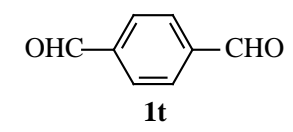

${ }^{\mathrm{a}}$ All products were identified by their physical and spectral data; ${ }^{\mathrm{b}}$ Isolated yields; ${ }^{\mathrm{C}}$ References for literature melting point. 
points and spectral data with their literature data.

\section{Conclusion}

We have described a potentially efficient, absolutely clean, and high yielding eco-friendly methodology, for the photochemical Knoevenagel condensation of various aromatic aldehydes with malononitrile catalyzed by aqueous starfruit juice. The present protocol devoid of any toxic catalysts, solvents or solid supports and may be considered as an excellent improvement over the existing methods.

\section{Experimental Section}

All reactions were run in dried glassware. Reagents were purchased (Spectrochem or SRL or LOBA) and used without further purification. Melting points were determined on a Kofler block and uncorrected. Reactions were irradiated in a $200 \mathrm{~W}$ tungsten lamp (Philips India Ltd). ${ }^{1} \mathrm{H}$ and ${ }^{13} \mathrm{C}$ NMR and spectra were obtained in $\mathrm{CDCl}_{3}$ or DMSO- $\mathrm{d}_{6}$ on a Bruker AV-300 (300 MHz) spectrometers using TMS as an internal standard. Analytical samples were dried in vacuo at room temperature. The carbon, hydrogen and nitrogen percentages in synthesized products were analyzed by Perkin-Elmer 2400 series II C, H, N analyzers. Thin layer chromatography was carried out on silica gel.

\subsection{Preparation of Aqueous Extract of Starfruit Juice}

The mature green starfruit were purchased from the local market. The starfruit were cut into pieces with the help of knife. The hard green material $(20 \mathrm{~g})$ was boiled with water $(50 \mathrm{ml})$, cooled and it was centrifuged using micro centrifuge (REMI RM-12C). The clear portion of the aqueous extract $(\mathrm{pH}=3.5)$ of the starfruit was used as catalyst for the reactions.

\subsection{General Procedure for Photochemical Knoevenagel Condensation Reaction}

Different aromatic aldehydes (1a-s) $(10 \mathrm{mmol})$ or $(\mathbf{1 t})$ (5 mmol), malononitrile (10 mmol), and aqueous starfruit juice $(5 \mathrm{ml}, \mathrm{pH}=3.5)$ were taken in a round bottomed flask and irradiated with a $200 \mathrm{~W}$ tungsten lamp (Philips India Ltd). The reaction time varied from 2 - 7 min monitored by TLC. Upon completion of the reaction, the reaction mixture was cooled and the crystalline products (3a-t) so obtained was filtered, washed with water and dried in vacuo. The Knoevenagel condensation products were isolated in excellent yields in essentially pure form.

\subsection{Spectral Data for Some Selected Compounds}

2-(3-Hydroxyphenylmethylene)malononitrile (3e): Yellow crystal, Yield: 95\%, mp. $165{ }^{\circ} \mathrm{C} ;{ }^{1} \mathrm{H}$ NMR $(300 \mathrm{MHz}$, DMSO-d ${ }_{6}$ ): $\delta 7.08$ (d, $\left.7.5 \mathrm{~Hz}, 1 \mathrm{H}\right), 7.35-7.44(\mathrm{~m}, 3 \mathrm{H}), 8.44$ (s, $\left.1 \mathrm{H}, \mathrm{H}-\mathrm{C}=\mathrm{C}\right), 10.12$ (s, $\left.1 \mathrm{H}, \mathrm{OH}\right)$; Anal. Calcd. for $\mathrm{C}_{10} \mathrm{H}_{6} \mathrm{~N}_{2} \mathrm{O}$, C, 70.58; $\mathrm{H}, 3.55$; N, 16.46\%, found C, 70.22; H, 3.87; N, 16.21\%.

2-(4-Benzoyloxyphenylmethylene)malononitrile (3n): Colorless crystal, Yield: $94 \%$, mp. $152{ }^{\circ} \mathrm{C}-154^{\circ} \mathrm{C} ;{ }^{1} \mathrm{H}$ NMR (300 MHz, CDCl $): \delta 7.43(\mathrm{~d}, 8.7 \mathrm{~Hz}, 2 \mathrm{H}), 7.54(\mathrm{t}, 7.5 \mathrm{~Hz}, 2 \mathrm{H}), 7.66-7.78(\mathrm{~m}, 1 \mathrm{H}), 7.78(\mathrm{~s}, 1 \mathrm{H}, \mathrm{H}-\mathrm{C}=\mathrm{C})$, 8.01 (d, $8.7 \mathrm{~Hz}, 2 \mathrm{H}), 8.20$ (d, $7.8 \mathrm{~Hz}, 2 \mathrm{H}) ;{ }^{13} \mathrm{C} \mathrm{NMR}\left(75 \mathrm{MHz}, \mathrm{CDCl}_{3}\right): \delta 82.54(=\mathrm{C}<), 112.49(\mathrm{CN}), 113.61$ (CN), 123.12, 128.41, 128.53, 128.75, 130.29 (-CH=), 132.37, 134.20, 155.56, 158.56, 164.24 (ester carbonyl); DEPT - 90 (75 MHz, $\left.\mathrm{CDCl}_{3}\right)$ : 123.11, 128.74, 130.28, 132.35, 134.18, 158.52; DEPT - 135 (75 MHz, $\left.\mathrm{CDCl}_{3}\right)$ : 123.11, 128.74, 130.28, 132.35, 134.18, 158.51; Anal. Calcd. for $\mathrm{C}_{17} \mathrm{H}_{10} \mathrm{~N}_{2} \mathrm{O}_{2}$, C, 74.45; H, 3.67; N, 10.21\%, found C, 74.11; H, 3.81; N, 10.43\%.

2-(4-Benzoyloxy-3-methoxyphenylmethylene)malono-nitrile (3o): Colorless crystal, Yield: 92\%, mp. $140^{\circ} \mathrm{C}$ $141^{\circ} \mathrm{C}$; ${ }^{1} \mathrm{H}$ NMR (300 MHz, CDCl $)$ ): $\delta 3.89$ (s, 3H, OMe), 7.34 (d, $\left.8.4 \mathrm{~Hz}, 1 \mathrm{H}\right), 7.43(\mathrm{dd}, 8.7$ and $1.8 \mathrm{~Hz}, 1 \mathrm{H})$, 7.53 (t, $7.5 \mathrm{~Hz}, 2 \mathrm{H}), 7.64-7.69$ (m, 1H), 7.74 (d, $1.8 \mathrm{~Hz}, 1 \mathrm{H}), 7.76$ (s, 1H, H-C=C), 8.20 (d, $8.7 \mathrm{~Hz}, 2 \mathrm{H})$;

Anal. Calcd. for $\mathrm{C}_{18} \mathrm{H}_{12} \mathrm{~N}_{2} \mathrm{O}_{3}$, C, 71.05; H, 3.97; N, 9.21\%, found C, 70.85; H, 4.02; N, 9.52\%.

2-(3,4-Methylenedioxyphenylmethylene)malononitrile (3p): Yellow crystal, Yield: $98 \%$, mp. $200^{\circ} \mathrm{C}-202^{\circ} \mathrm{C}$; ${ }^{1} \mathrm{H}$ NMR (300 MHz, $\mathrm{CDCl}_{3}$ ): $\delta 6.12$ (s, 2H, -O-CH $-\mathrm{O}-$ ), 6.93 (d, $\left.8.1 \mathrm{~Hz}, 1 \mathrm{H}\right), 7.32$ (dd, 8.1 and $1.5 \mathrm{~Hz}, 1 \mathrm{H}$ ), 7.59 (s, $1 \mathrm{H}, \mathrm{H}-\mathrm{C}=\mathrm{C}$ ), 7.60 (s, 1H); Anal. Calcd. for $\mathrm{C}_{11} \mathrm{H}_{6} \mathrm{~N}_{2} \mathrm{O}_{2}$, C, 66.67; H, 3.05; N, 14.14\%, found C, 67.01; H, $3.21 ; \mathrm{N}, 14.32 \%$. 
2-[\{p-3, 3'-Bis(2-methylindolyl)methyl\}phenyl-methylene]malononitrile (3s): Pale-yellow crystal, Yield: 78\%, mp. $320^{\circ} \mathrm{C}-322^{\circ} \mathrm{C} ;{ }^{1} \mathrm{H}$ NMR (300 MHz, $\mathrm{CDCl}_{3}$ ): $\delta 2.09$ (s, 6H, Me), 6.04 (s, $\left.1 \mathrm{H}, \mathrm{Ar}-\mathrm{CH}\right), 6.84-6.93(\mathrm{~m}, 4 \mathrm{H})$, 7.06 (t, $6.9 \mathrm{~Hz}, 2 \mathrm{H}), 7.28$ (d, $9.0 \mathrm{~Hz}, 2 \mathrm{H}), 7.44$ (d, $8.1 \mathrm{~Hz}, 2 \mathrm{H}), 7.72$ (s, 1H, H-C=C), 7.80 (d, $8.7 \mathrm{~Hz}, 2 \mathrm{H}), 7.80$ (br. s, 2H, NH); Anal. Calcd. for $\mathrm{C}_{29} \mathrm{H}_{22} \mathrm{~N}_{4}, \mathrm{C}, 81.67 ; \mathrm{H}, 5.20$; N, 13.14\%, found C, 81.33; H, 5.40; N, $13.25 \%$.

p-Bis-2-(phenylmethylene)malononitrile (3t): White crystal, Yield: $95 \%$, mp. $300{ }^{\circ} \mathrm{C} ;{ }^{1} \mathrm{H}$ NMR $(300 \mathrm{MHz}$, DMSO-d $\mathrm{d}_{6}$ ): $\delta 8.09$ (s, 4H), 8.63 (s, 2H, H-C=C); ${ }^{13} \mathrm{C}$ NMR (75 MHz, DMSO-d $\left.\mathrm{d}_{6}\right): \delta 84.71(=\mathrm{C}<$ ), $112.14(\mathrm{CN})$, $113.80(\mathrm{CN}), 130.83(-\mathrm{CH}=), 135.32$ (aromatic quarternary), 159.80 (aromatic -CH=); DEPT - 90 (75 MHz, DMSO-d ${ }_{6}$ ): 130.83, 159.81; DEPT - 135 (75 MHz, DMSO-d $\mathrm{d}_{6}$ ): 130.84, 159.81; Anal. Calcd. for $\mathrm{C}_{14} \mathrm{H}_{6} \mathrm{~N}_{4}$, C, 73.04; H, 2.63; N, 24.34\%, found C, 72.95, H, 2.76; N, 24.45\%.

\section{Acknowledgements}

Financial assistance from the UGC Minor Research Project No. PSW-130/11-12 (ERO), New Delhi, India is gratefully acknowledged.

\section{References}

[1] Knoevenagel, E. (1894) Uebereine Darstellungsweise der Glutarsaure. Berichte der Deutschen Chemischen Gesellschaft, 2, 2345-2346. http://dx.doi.org/10.1002/cber.189402702229

[2] Knoevenagel, E. (1898) Condensationen zwisschen malonester und aldehyden unter dem einfluss von ammoniak und organischen aminen. Chemische Berichte, 31, 2585-2595. http://dx.doi.org/10.1002/cber.18980310307

[3] Jones, G. (1967) The Knoevenagel Condensation. Organic Reactions, 15, 204-599.

[4] Tietze, L.F. and Beifuss, U. (1991) The Knoevenagel Reaction. In: Trost, B.M., Ed., Comprehensive Organic Synthesis, Pergamon Press, Oxford, 341-394. http://dx.doi.org/10.1016/B978-0-08-052349-1.00033-0

[5] Yu, N., Aramini, J.M., Germann, M.W. and Huang, Z. (2000) Reactions of Salicylaldehydes with Alkyl Cyanoacetates on the Surface of Solid Catalysts: Synthesis of 4H-Chromene Derivatives. Tetrahedron Letters, 41, 6993-6996. http://dx.doi.org/10.1016/S0040-4039(00)01195-3

[6] Gallos, J., Discordia, R.P., Crispino, G.A., Li, J., Grosso, J.A., Polniaszek, V. and True, V.C. (2003) A Mild and Efficient Synthesis of 4-Aryl-Quinolin-2(1H)-Ones via a Tandem Amidation/Knoevenagel Condensation of 2-AminoBenzophenones with Esters or Lactones. Tetrahedron Letters, 44, 4271-4273. http://dx.doi.org/10.1016/S0040-4039(03)00889-X

[7] Xing, C. and Zhu, S. (2004) Unexpected Formation of Tetrasubstituted 2,3-Dihydrofurans from the Reactions of $\beta$ Keto Polyfluoroalkanesulfones with Aldehydes. Journal of Organic Chemistry, 69, 6486-6488.

http://dx.doi.org/10.1021/jo049317y

[8] Tietze, L.F. and Rackelmann, N. (2004) Domino Reactions in the Synthesis of Heterocyclic Natural Products and Analogs. Pure and Applied Chemistry, 76, 1967-1983. http://dx.doi.org/10.1351/pac200476111967

[9] Vijender, M., Kishor, P. and Satyanarayana, B. (2008) Zirconium Tetrachloride- $\mathrm{SiO}_{2}$ Catalyzed Knoevenagel Condensation: A Simple and Efficient Protocol for the Synthesis of Substituted Electrophilic Alkenes. Arkivoc, 2008, 122-128. http://dx.doi.org/10.3998/ark.5550190.0009.d14

[10] Rao, P.S. and Venkataratnam, R.V. (1991) Zinc Chloride as a New Catalyst for Knoevenagel Condensation. Tetrahedron Letters, 32, 5821-5822. http://dx.doi.org/10.1016/S0040-4039(00)93564-0

[11] Prajapati, D. and Sandhu, J.S. (1993) Cadmium Iodide as a New Catalyst for Knoevenagel Condensation. Journal of the Chemical Society, Perkin Transactions 1, 1, 739-740. http://dx.doi.org/10.1039/p19930000739

[12] Lehnert, W. (1970) Verbesserte Variante der Knoevenagel-Kondensation Mit $\mathrm{TiCl}_{4} / \mathrm{THF} /$ Pyridine (I). Alkyliden-und Arylidenmalonester bei 0 - $25^{\circ} \mathrm{C}$. Tetrahedron Letters, 11, 4723-4724. http://dx.doi.org/10.1016/S0040-4039(00)89377-6

[13] Dai, G., Shi, D., Zhou, L. and Huaxue, Y. (1995) Knoevenagel Condensation Catalysed by Potassium Fluoride/Alumina. Chinese Journal of Applied Chemistry, 12, 104-108.

[14] Gill, C., Pandhare, G., Raut, R., Gore, V. and Gholap, S. (2008) Knoevenagel Condensation: A Simple and Efficient Protocol of Electrophilic Alkenes Catalyzed by Anhydrous Ferric Sulphate with Remarkable Reusability. Bulletin of the Catalysis Society of India, 7, 153-157.

[15] Bogdal, D. (1998) Coumarins: Fast Synthesis by Knoevenagel Condensation under Microwave Irradiation. Journal of Chemical Research (Synopsis), 8, 468-469. http://dx.doi.org/10.1039/a801724g

[16] Kumar, H.M.S., Reddy, B.V.S., Anjaneyulu, S. and Yadav, J.S. (1998) Non Solvent Reaction: Ammonium Acetate 
Catalyzed Highly Convenient Preparation of Trans-Cinnamic Acid. Synthetic Communications, 28, 3811-3815. http://dx.doi.org/10.1080/00397919808004934

[17] Kumar, H.M.S., Reddy, B.V.S., Reddy, P.T., Srinivas, D. and Yadav, J.S. (2000) Silica Gel Catalyzed Preparation of Cinnamic Acid under Microwave Irradiation. Organic Preparations Procedures International, 32, 81-83. http://dx.doi.org/10.1080/00304940009356750

[18] Mogilaiah, K. and Reddy, C.S. (2003) An Efficient Friedlander Condensation Using Sodium Fluoride as Catalyst in the Solid State. Synthetic Communications, 33, 3131-3134. http://dx.doi.org/10.1081/SCC-120023427

[19] Mallouk, S., Bougrin, K., Laghzizil, A. and Benhida, R. (2010) Microwave-Assisted and Efficient Solvent-Free Knoevenagel Condensation. A Sustainable Protocol Using Calcium Hydroxyapatite as Catalyst. Molecules, 15, 813-823.

[20] Bhuiyan, M.M.H., Hossain, M.I., Alam, M.A. and Mahmud, M.M. (2012) Microwave Assisted Knoevenagel Condensation: Synthesis and Antimicrobial Activities of Some Arylidene-Malononitriles. Chemistry Journal, 2, 30-36.

[21] McNulty, J., Steere, J.A. and Wolf, S. (1998) The Ultrasound Promoted Knoevenagel Condensation of Aromatic Aldehydes. Tetrahedron Letters, 39, 8013-8016. http://dx.doi.org/10.1016/S0040-4039(98)01789-4

[22] Palmisano, G., Tibiletti, F., Penoni, A., Colombo, F., Tollari, S., Garella, D., Tagliapietra, S. and Cravotto, G. (2011) Ultrasound-Enhanced One-Pot Synthesis of 3-(Het)arylmethyl-4-hydroxycoumarins in Water. Ultrasonics Sonochemistry, 18, 652-660. http://dx.doi.org/10.1016/j.ultsonch.2010.08.009

[23] Pratap, U.R., Jawale, D.V., Waghmare, R.A., Lingampalle, D.L. and Mane, R.A. (2011) Synthesis of 5-arylidene-2, 4-thiazolidinediones by Knoevenagel Condensation Catalyzed by Baker's Yeast. New Journal of Chemistry, 35, 49-51. http://dx.doi.org/10.1039/c0nj00691b

[24] Wang, C.H., Guan, Z. and He, Y.H. (2011) Biocatalytic Domino Reaction: Synthesis of 2H-1-benzopyran-2-one Derivatives Using Alkaline Protease from Bacillus licheniformis. Green Chemistry, 13, 2048-2054. http://dx.doi.org/10.1039/c0gc00799d

[25] Xia, Y., Yang, Z.Y., Brossi, A. and Lee, K.H. (1999) Asymmetric 'Solid-Phase Synthesis of (3`R,4`R)-Di-O-cis-acyl 3-Carboxyl Khellactones. Organic Letters, 1, 2113-2115. http://dx.doi.org/10.1021/ol991168w

[26] Guo, G., Arvanitis, E.A., Pottorf, R.S. and Player, M.P. (2003) Solid-Phase Synthesis of a Tyrphostin Ether Library. Journal of Combinatorial Chemistry, 5, 408-413. http://dx.doi.org/10.1021/cc030003i

[27] Ying, A.G., Liu, L., Wu, G.F., Chen, X.Z., Ye, W.D., Chen, J.H. and Zhang, K.Y. (2009) Knoevenagel Condensation Catalyzed by DBU Brönsted Ionic Liquid without Solvent. Chemical Research in Chinese Universities, 25, 876-881.

[28] Khan, F.A., Dash, F.J., Satapathy, R. and Upadhyay, S.K. (2004) Hydrotalcite Catalysis in Ionic Medium: A Recyclable Reaction System for Heterogeneous Knoevenagel and Nitroaldol Condensation. Tetrahedron Letters, 45, 30553058. http://dx.doi.org/10.1016/j.tetlet.2004.02.103

[29] Verdia, P., Santamarta, F. and Tojo, E. (2011) Knoevenagel Reaction in [MMIm][MSO $\left.{ }_{4}\right]$ : Synthesis of Coumarins. Molecules, 16, 4379-4388. http://dx.doi.org/10.3390/molecules16064379

[30] Hu, Y., Chen, J., Le, Z.G. and Zheng, Q.G. (2005) Organic Reactions in Ionic Liquids: Ionic Liquids Ethylammonium Nitrate Promoted Knoevenagel Condensation of Aromatic Aldehydes with Active Methylene Compounds. Synthetic Communications, 35, 739-744. http://dx.doi.org/10.1081/SCC-200050380

[31] Xin, X., Guo, X., Duan, H., Lin, Y. and Sun, H. (2007) Efficient Knoevenagel Condensation Catalyzed by Cyclic Guanidinium Lactate Ionic Liquid as Medium. Catalysis Communications, 8, 115-117. http://dx.doi.org/10.1016/j.catcom.2006.05.034

[32] Santamarta, F., Verdía, P. and Tojo, E. (2008) A Simple, Efficient and Green Procedure for Knoevenagel Reaction in [MMIm] $\left[\mathrm{MSO}_{4}\right]$ Ionic Liquid. Catalysis Communications, 9, 1779-1781.

[33] Yue, C., Mao, A., Wei, Y. and Lü, M. (2008) Knoevenagel Condensation Reaction Catalyzed by Task-Specific Ionic Liquid under Solvent-Free Conditions. Catalysis Communications, 9, 1571-1574. http://dx.doi.org/10.1016/j.catcom.2008.01.002

[34] Bigi, F., Conforti, M.L., Maggi, R., Piccinno, A. and Sartori, G. (2000) Clean Synthesis in Water: Uncatalysed Preparation of Ylidenemalonitriles. Green Chemistry, 2, 101-103. http://dx.doi.org/10.1039/b001246g

[35] Wang, S., Ren, Z., Cao, W. and Tong, W. (2001) The Knoevenagel Condensation of Aromatic Aldehydes with Malononitrile or Ethyl Cyanoacetate in the Presence of CTMAB in Water. Synthetic Communications, 31, 673-677. http://dx.doi.org/10.1081/SCC-100103255

[36] Oskooie, H.A., Heravi, M.M., Derikvand, F., Khorasani, M. and Bamoharram, F.F. (2006) On Water: An Efficient Knoevenagel Condensation Using 12-Tungstophoric Acid as a Reusable Green Catalyst. Synthetic Communications, 36, 2819-2823. http://dx.doi.org/10.1080/00397910600770631

[37] Pasha, M.A. and Manjula, K. (2011) Lithium Hydroxide: A Simple and an Efficient Catalyst for Knoevenagel Condensation under Solvent-Free Grindstone Method. Journal of Saudi Chemical Society, 15, 283-286. 
http://dx.doi.org/10.1016/j.jscs.2010.10.010

[38] Rong, L., Li, X., Wang, H., Shi, D., Tu, S. and Zhuang, Q. (2006) Efficient Green Procedure for the Knoevenagel Condensation under Solvent-Free Conditions. Synthetic Communications, 36, 2407-2412. http://dx.doi.org/10.1080/00397910600640289

[39] Ren, Z., Cao, W. and Tong, W. (2002) The Knoevenagel Condensation Reaction of Aromatic Aldehydes with Malononitrile by Grinding in the Absence of Solvents and Catalysts. Synthetic Communications, 32, 3475-3479. http://dx.doi.org/10.1081/SCC-120014780

[40] Okkerse, C. and van Bekkun, H. (1999) From Fossil to Green. Green Chemistry, 1, 107-114. http://dx.doi.org/10.1039/a809539f

[41] Anastas, P.T. and Warner, J.C. (2000) Green Chemistry: Theory and Practice. Oxford University Press, New York.

[42] Hoffmann, N. (2008) Photochemical Reactions as Key Steps in Organic Synthesis. Chemical Reviews, 108, $1052-1103$. http://dx.doi.org/10.1021/cr0680336

[43] Fagnoni, M., Dondi, D., Ravelli, D. and Albini, A. (2007) Photocatalysis for the Formation of the C-C Bond. Chemical Reviews, 107, 2725-2756. http://dx.doi.org/10.1021/cr068352x

[44] Ghosh, S. and Das, J. (2011) A Novel Photochemical Wittig Reaction for the Synthesis of 2-Aryl/Alkylbenzofurans. Tetrahedron Letters, 52, 1112-1116. http://dx.doi.org/10.1016/j.tetlet.2010.12.104

[45] Ghosh, S., Das, J. and Chattopadhyay, S. (2011) A Novel Light Induced Knoevenagel Condensation of Meldrum’s Acid with Aromatic Aldehydes in Aqueous Ethanol. Tetrahedron Letters, 52, 2869-2872. http://dx.doi.org/10.1016/j.tetlet.2011.03.123

[46] Ghosh, S., Das, J. and Saikh, F. (2012) A New Synthesis of 2-Aryl/Alkylbenzofurans by Visible Light Stimulated Intermolecular Sonogashira Coupling and Cyclization Reaction in Water. Tetrahedron Letters, 53, 5883-5886. http://dx.doi.org/10.1016/j.tetlet.2012.08.078

[47] Ghosh, S., Saikh, F., Das, J. and Pramanik, A.K. (2013) Hantzsch 1,4-Dihydropyridine Synthesis in Aqueous Ethanol by Visible Light. Tetrahedron Letters, 54, 58-62. http://dx.doi.org/10.1016/j.tetlet.2012.10.079

[48] Li, C. and Chen, L. (2006) Organic Chemistry in Water. Chemical Society Reviews, 35, 68-82. http://dx.doi.org/10.1039/b507207g

[49] Breslow, R. (1991) Hydrophobic Effects on Simple Organic Reactions in Water. Accounts of Chemical Research, 24, 159-164. http://dx.doi.org/10.1021/ar00006a001

[50] Ramesh, E. and Raghunathan, R. (2009) Microwave-Assisted K-10 Montmorillonite Clay-Mediated Knoevenagel Hetero-Diels-Alder Reactions: A Novel Protocol for the Synthesis of Polycyclic Pyrano[2,3,4-kl]xanthenes Derivatives. Synthetic Communications, 39, 613-625. http://dx.doi.org/10.1080/00397910802417825

[51] Habibi, D. and Marvi, O. (2006) Montmorillonite KSF and Montmorillonite K-10 Clays as Efficient Catalysts for the Solventless Synthesis of Bismaleimides and Bisphthalimides Using Microwave Irradiation. Arkivoc: Online Journal of Organic Chemistry, 2006, 8-15. http://dx.doi.org/10.3998/ark.5550190.0007.d02

[52] Chakrabarty, M., Mukherjee, A., Arima, S., Harigaya, Y. and Pilet, G. (2009) Expeditious Reaction of Ninhydrin with Active Methylene Compounds on Montmorillonite K10 Clay. Monatshefte Für Chemie_Chemical Monthly, 140, 189197. http://dx.doi.org/10.1007/s00706-008-0066-6

[53] Wada, S. and Suzuki, H. (2003) Calcite and Fluorite as Catalyst for the Knoevenagel Condensation of Malononitrile and Methyl Cyanoacetate under Solvent-Free Conditions. Tetrahedron Letters, 44, 399-401. http://dx.doi.org/10.1016/S0040-4039(02)02431-0

[54] Zahouily, M., Mounir, B., Charki, H., Mezdar, A., Bahlaouan, B. and Ouammou, M. (2006) Investigation of the Basis Catalytic Activity of Natural Phosphates in the Michael Condensation. Arkivoc: Online Journal of Organic Chemistry, 2006, 178-186. http://dx.doi.org/10.3998/ark.5550190.0007.d19

[55] Zahouily, M., Bahlaouan, B., Rayadh, A. and Sebti, S. (2004) Natural Phosphates and Potassium Fluoride Doped Natural Phosphate: Efficient Catalyst for the Construction of a Carbon-Nitrogen Bond. Tetrahedron Letters, 45, 41354138. http://dx.doi.org/10.1016/j.tetlet.2004.03.164

[56] Sebti, S., Smahi, A. and Solly, A. (2002) Natural Phosphate Doped with Potasiuum Fluoride and Modified with Sodium Nitrate: Efficient Catalysts for the Knoevenagel Condensation. Tetrahedron Letters, 43, 1813-1815. http://dx.doi.org/10.1016/S0040-4039(02)00092-8

[57] Riadi, Y., Mamouni, R., Azzalou, R., Boulahjar, R., Abrouki, Y., El Haddad, M., Routier, S., Guillaumet, G. and Lazar, S. (2010) Animal Bone Meal as an Efficient Catalyst for Crossed-Aldol Condensation. Tetrahedron Letters, 51, 67156717. http://dx.doi.org/10.1016/j.tetlet.2010.10.056

[58] Deshmukh, M.B., Patil, S.S., Jadhav, S.D. and Pawar, P.B. (2012) Green Approach for Knoevenagel Condensation of Aromatic Aldehydes with Active Methylene Group. Synthetic Communications, 42, 1177-1183. 
http://dx.doi.org/10.1080/00397911.2010.537423

[59] Patil, S., Jadhav, S.D. and Deshmuk, M.B. (2011) Natural Acid Catalyzed Multi-Component Reaction as a Green Approach. Archives of Applied Science Research, 3, 203-208.

[60] Sachdeva, H., Saroj, R., Khaturia, S. and Dwivedi, D. (2013) Environ-Economic Synthesis and Characterization of Some New 1,2,4-Triazole Derivative as Organic Fluorescent Materials and Potent Fungicidal Agents. Organic Chemistry International, 2013, Article ID 659107.

[61] Patil, S., Jhadav, S.D. and Patil, U.P. (2012) Natural Acid Catalyzed Synthesis of Schiff Base under Solvent-Free Condition: As a Green Approach. Archives of Applied Science Research, 4, 1074-1078.

[62] Pal, R., Khasnobis, S. and Sarkar, T. (2013) First Application of Fruit Juice of Citrus limon for Facile and Green Synthesis of Bis- and Tris(indolyl)methanes in Water. Chemistry Journal, 3, 7-12.

[63] Pal, R. (2013) Microwave-Assisted Eco-Friendly Synthesis of Bis-, Tris(indolyl)methanes and Synthesis of Dibis(indolyl)methanes Catalyzed by Fruit Juice of Citrus limon under Solvent-Free Conditions. IOSR Journal of Applied Chemistry, 3, 1-8. http://dx.doi.org/10.9790/5736-0340108

[64] Pal, R. (2013) New Greener Alternative for Biocondensation of Aldehydes and Indoles Using Lemon Juice: Formation of Bis-, Tris-, and Tetraindoles. International Journal of Organic Chemistry, 3, 136-142. http://dx.doi.org/10.4236/ijoc.2013.32015

[65] Patil, S., Jadhav, S.D. and Mane, S. (2011) Pineapple Juice as a Natural Catalyst: An Excellent Catalyst for Biginelli Reaction. Journal of Organic Chemistry, 1, 125-131.

[66] Patil, S., Jadhav, S.D. and Deshmukh, M.B. (2013) Eco-Friendly and Economic Method for Knoevenagel Condensation by Employing Natural Catalyst. Indian Journal of Chemistry, 52B, 1172-1175.

[67] Fonseca, A.M., Monte, F.J., de Oliveira, M.C.F., de Mattos, M.C.M., Cordell, G.A., Braz-Filho, R. and Lemos, T.L.G. (2009) Coconut Water (Cocos nucifera L.) —A New Biocatalyst System for Organic Synthesis. Journal of Molecular Catalysis B: Enzymatic, 57, 78-82. http://dx.doi.org/10.1016/j.molcatb.2008.06.022

[68] Mote, K., Pore, S., Rashinkar, G., Kambale, S., Kumbhar, A. and Salunkhe, R. (2010) Acacia Concinna Pods: As a Green Catalyst for Highly Efficient Synthesis of Acylation of Amines. Archives of Applied Science Research, 2, 74-80.

[69] Pore, S., Rashimkar, G., Mote, K. and Salunkhe, R. (2010) Aqueous Extract of the Pericarp of Sapindus trifoliatus Fruits: A Novel ‘Green’ Catalyst for the Aldimine Synthesis. Chemistry and Biodiversity, 7, 1796-1800. http://dx.doi.org/10.1002/cbdv.200900272

[70] Pal, R. (2013) A Convenient, Rapid and Eco-Friendly Synthesis of Bis-, Tris(indolyl)methanes and Synthesis of Tetraindolyl Compounds Catalyzed by Tamarind Juice under Microwave Irradiation. International Journal of Chemtech Applications, 2, 26-40.

[71] Pal, R. (2014) Visible Light Induced Knoevenagel Condensation: A Clean and Efficient Protocol Using Aqueous Fruit Extract of Tamarindus indica as Catalyst. International Journal of Advanced Chemistry, 2, 27-33.

[72] Sripanidkulchai, B., Tattawasart, U., Laupattarakasem, P. and Wongpanich, V. (2002) Anti-Inflammatory and Bactericidal Properties of Selected Indigeneous Medicinal Plants Used for Dysuria. Thai Journal of Pharmaceutical Sciences, 26, 33-38.

[73] Morton, J.F. (1987) Carambola. In: Dowling, C.F., Ed., Fruits of Warm Climates, Flair Books, Miami, 125-128.

[74] Dasgupta, P., Chakraborty, P. and Bala, N.N. (2013) Averrhoa carambola: An Updated Review. International Journal of Pharma Research \& Review, 2, 54-63. 
Scientific Research Publishing (SCIRP) is one of the largest Open Access journal publishers. It is currently publishing more than 200 open access, online, peer-reviewed journals covering a wide range of academic disciplines. SCIRP serves the worldwide academic communities and contributes to the progress and application of science with its publication.

Other selected journals from SCIRP are listed as below. Submit your manuscript to us via either submit@scirp.org or Online Submission Portal.
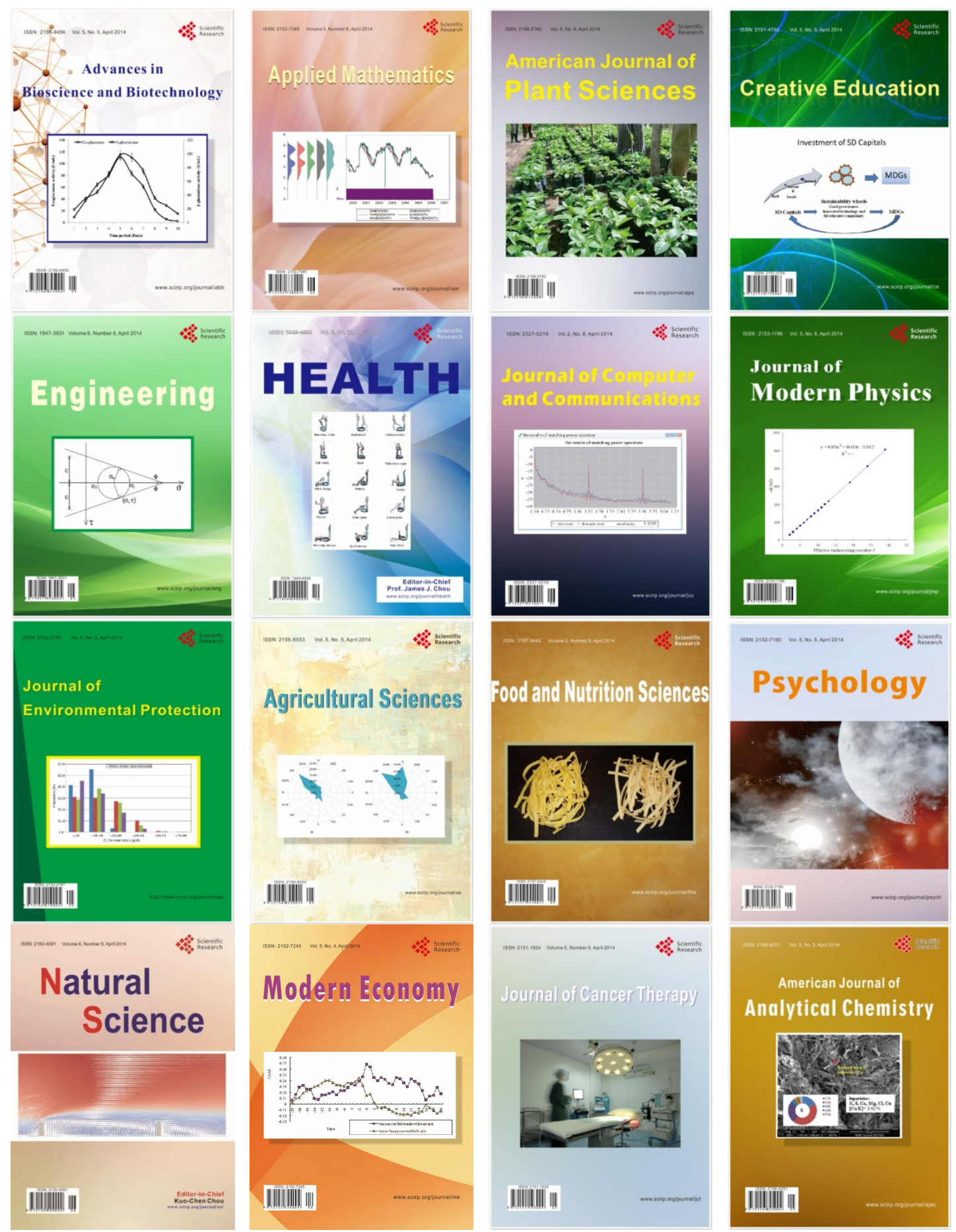